\title{
Barreiras à implementação da logística reversa e do Green supply Chain Manasement (GSCM)
}

\section{Barriers to implementation of reverse logistics and Green Supply Chain Management (GSCM)}

\section{Barreras a la implementación de la logística reversa y del Green Supply Chain Management (GSCM)}

\author{
Márcia Maria Penteado MarchesinI \\ Professora Adjunta da Universidade Federal do $A B C$ (UFABC) \\ FABIANE LETÍCIA LIZARELLI \\ Professora Adjunta da Universidade Federal de São Carlos
}

REsumo O GSCM (Green Supply Chain Management) surgiu para integrar a preocupação ambiental com a SCM e requer que, além de atividades da cadeia de suprimentos direta adequadas ambientalmente, que tais atividades sejam estendidas para incluir cadeias de suprimentos reversas. No "fechamento do ciclo" da cadeia, materiais em seus finais de vida são trazidos de volta ao sistema para serem recuperados, sendo a Logística Reversa somente parte do conceito de GSCM. A literatura indica os benefícios da implantação de práticas do GSCM, mas o meio empresarial ainda tem muitos desafios para estabelecer cadeias de suprimentos verdadeiramente sustentáveis, tendo que lidar com barreiras de implementação. As empresas precisam remover essas barreiras, mas se observa que ainda há pouca avaliação sistematizada da literatura sobre essa questão. Diante dessa lacuna de pesquisa, o objetivo do artigo é levantar na literatura, por meio da Revisão Bibliográfica Sistemática, as barreiras para a implementação efetiva da Logística Reversa e do GSCM. Por intermédio desse método, foram encontradas 59 barreiras à implementação da LR e/ou GSCM, enquadradas em sete diferentes categorias: gestão, financeiras, stakeholders, infraestrutura e tecnologia, ciclo de vida, conhecimento e cultura, revelando a grande variedade de fatores que podem dificultar a implementação de ações ambientalmente sustentáveis nas organizações.

Palavras-chave: Sustentabilidade ambiental. Gestão da Cadeia de Suprimentos Verde. Logística Reversa. Barreiras à implementação. Revisão Bibliográfica Sistemática. 
Aвstract The GSCM (Green Supply Chain Management) has emerged to integrate environmental concern with SCM and requires that in addition to direct supply chain activities be environmentally appropriate, such activities should be extended to include reverse supply chains. In the "cycle closing" of chain, materials in its end of life are brought back to the system to be recovered, and being Reverse Logistics only part of the concept of GSCM. The literature indicates the benefits of implementing GSCM practices, but the business environment still has many challenges to establish truly sustainable supply chains, having to deal with implementation barriers. Companies need to remove these barriers, but notes that there is little systematic evaluation of the literature on this issue. Given this research gap, the paper aims to raise in the literature, through the Systematic Literature Review, the barriers to effective implementation of Reverse Logistics and GSCM. Through this method, it was found 59 barriers to the implementation of the Reverse Logistics and/or GSCM, framed in seven different categories: management, financial, stakeholders, infrastructure and technology, life cycle, knowledge and culture, revealing the wide variety of factors that may hinder the implementation of environmentally sustainable actions in the organizations.

Keywords: Environmental sustainability. Green Supply Chain Management. Reverse LoGistics. BARriers to implementation. SyStematic Literature REVIEW.

Resumen La GSCM (Green Supply Chain Management) surgió para integrar la preocupación ambiental con la SCM y requiere que, además de actividades de la cadena de suministros directa adecuadas ambientalmente, que tales actividades se extiendan para incluir cadenas de suministros reversas. En el "cierre del ciclo" de la cadena, materiales en sus finales de vida son traídos de vuelta al sistema para ser recuperados, siendo la Logística Reversa solamente parte del concepto de GSCM. La literatura indica los beneficios de la implantación de prácticas del GSCM, pero el medio empresarial todavía tiene muchos desafíos para establecer cadenas de suministros verdaderamente sostenibles, teniendo que lidiar con barreras de implementación. Las empresas necesitan eliminar esas barreras, pero se observa que todavía hay poca evaluación sistematizada de la literatura sobre esa cuestión. Ante esta laguna de investigación, el objetivo del artículo es levantar en la literatura, por medio de la Revisión Bibliográfica Sistemática, las barreras para la implementación efectiva de la Logística Reversa y del GSCM. Por medio de este método, se encontraron 59 barreras a la implementación de la LR y / o GSCM, enmarcadas en siete diferentes categorías: gestión, financieras, stakeholders, infraestructura y tecnología, ciclo de vida, conocimiento y cultura, revelando la gran variedad de factores que pueden dificultando la implementación de acciones ambientalmente sostenibles en las organizaciones. Palabras clave: Sostenibilidad ambiental. Gestión de la cadena de suministros verdes. Logística Reversa. Barreras a la implementación. Revisión Bibliográfica Sistemática

\section{INTRODUÇÃO}

A propagação da consciência ambiental na sociedade e as regulações ambientais cada vez mais restritivas elaboradas pelos governos pressionam as indústrias e outras empresas 
da cadeia de suprimentos na adoção de esforços visando reduzir seus impactos ambientais. Assim, o aumento da sensibilização para a proteção ambiental em todo o mundo exerce forte pressão sobre as empresas, as quais devem ter responsabilidade no papel que exercem sobre a sociedade e sobre a minimização dos impactos ambientais gerados por suas atividades (MATHIYAZHAGAN et al., 2013).

No Brasil, a importância da LR tem aumentado em decorrência da introdução da Política Nacional de Resíduos Sólidos (PNRS), criada em 2010, e por questões econômicas, como o valor recuperado dos produtos utilizados, que implica melhoria das condições sociais dos envolvidos com a LR, impulsionando também o marketing verde (BOUZON; GOVINDAN; RODRIGUEZ, 2015). A PNRS no Brasil e as diretrizes criadas em 2011 pela OECD (Organisation for Economic Co-operation and Development) são exemplos de regulações que instituem a necessidade de as organizações e suas cadeias de suprimentos se adequarem à sustentabilidade.

A PNRS foi instituída pelo governo brasileiro em 2010, por meio da Lei No. 12.305/2010, sendo um dos seus princípios a "responsabilidade compartilhada pelo ciclo de vida dos produtos". Tal responsabilidade consiste nas atribuições individualizadas e encadeadas dos fabricantes, importadores, distribuidores e comerciantes, dos consumidores e dos titulares dos serviços públicos de limpeza urbana e de manejo dos resíduos sólidos. Assim, todas as organizações da cadeia de suprimentos têm responsabilidade pelos impactos ambientais causados pelo ciclo de vida dos produtos e seus resíduos. A PNRS (2010) propõe a seguinte ordem de prioridade para as diferentes destinações dos resíduos: prevenção (não geração dos resíduos), redução, valorização (reutilização, remanufatura, reciclagem) e eliminação (tratamento dos resíduos sólidos e disposição final ambientalmente adequada dos rejeitos).

Rauer e Kaufmann (2015) salientam que as Diretrizes da OECD para Empresas Multinacionais (OECD, 2011) torna obrigatória a implementação de práticas sustentáveis da SCM além dos limites de uma única organização, buscando a gestão responsável da cadeia de suprimentos.

Conforme OECD (2011, 2016), não devem existir contradições entre as atividades das empresas multinacionais e o desenvolvimento sustentável: as empresas devem contribuir para o progresso econômico, social e ambiental, de modo a alcançar o desenvolvimento sustentável. No contexto da cadeia de suprimentos, há a responsabilidade da empresa sobre suas cadeias e, se uma empresa identifica riscos relativos a impactos adversos, ela deve tomar providências para cessar tais impactos ou já preveni-los de imediato.

Sarkis (2012) aponta que primeiro a questão da preservação ambiental surgiu dentro das empresas de forma reativa diante de pressões regulatórias e de legislação, para depois se tornar proativa visando criar vantagem competitiva organizacional a partir de uma forte imagem corporativa organizacional. Em seguida, diante da necessidade de criar relacionamentos interorganizacionais, surgiu a SCM (Supply Chain Management ou Gestão da Cadeia de Suprimentos), para gerar vantagem competitiva a partir da gestão da rede de fornecedores e clientes. O GSCM (Green Supply Chain Management) desponta em um terceiro momento para integrar a preocupação ambiental com a SCM. Nesse contexto, empresas com imagem e reputação green e que produzem produtos green tendem a adotar o GSCM, de modo a adequar suas cadeias de suprimentos ao conceito de sustentabilidade ambiental/ecológica (RAUER; KAUFMANN, 2015). 
A cadeia de suprimentos tem sido tradicionalmente definida como um processo integrado de uma única direção em que matérias-primas são convertidas em produtos finais, que são entregues aos clientes finais. Mas Beamon (1999) aponta que a cadeia de suprimentos verde ou ambientalmente correta necessita da extensão da cadeia tradicional com o objetivo de considerar todos os efeitos ambientais imediatos e eventuais de todos os produtos e processos ao longo de todo o ciclo de vida. Isso porque todos os elos de uma cadeia de suprimentos podem acarretar impactos ambientais (desde a extração das matérias-primas até a venda do produto), bem como, as etapas subsequentes de uso dos produtos e de disposição final dos mesmos.

O GSCM surgiu na década de 2000 como uma área com foco popular dentro dos aspectos tradicionais da SCM (MUDULIA et al., 2013a). O GSCM foi desenvolvido a partir de duas origens primárias:

a) Abordagem da Avaliação do Ciclo de Vida (LCA, Life Cycle Assessment);

b) Integração das Questões Ambientais aos processos e práticas da SCM.

O GSCM integra conceitos ecológicos com os de SCM e suas atividades tradicionais de cadeia de suprimentos, de modo a minimizar o uso de recursos (materiais, água e energia) e reduzir impactos adversos das atividades da cadeia de suprimentos sobre o meio ambiente, melhorando ou preservando-o (MUDULI et al., 2013a).

A literatura indica que houve variação na terminologia usada para o conceito de GSCM: Green Purchasing and Procurement, Environmental Purchasing, Green Logistics, Environmental Logistics, Supply and demand sustainability, Corporate social responsability networks, supply chain environmental management, sustainable supply chain management e sustainable supply network management (SARKIS, 2012; RAUER; KAUFMANN, 2015). Sendo assim, nota-se certa confusão entre os termos relacionados à cadeia de suprimentos sustentável e à logística sustentável, apesar de que a literatura (CSCMP, 2016; LAMBERT; GARCÍA-DASTUGUE; CROXTON, 2008; LAMBERT, 2008; BOWERSOX et al., 2014; LAMBERT; COOPER, 2000; KAUFFMAN, 2002) afirma que SCM e logística são termos considerados diferentes, sendo a logística somente parte da SCM.

Não existe clareza a respeito das diferenças entre os termos relativos à cadeia de suprimentos sustentável ou verde, logística sustentável ou verde e logística reversa. Tal confusão deriva do fato de que o GSCM e a LR são identificados na literatura como conceitos relativamente novos e cujas áreas ainda estão em desenvolvimento, sendo definidos de forma ampla. O GSCM é um campo ainda emergente, estando em evolução e possui muitas dimensões ou elementos (SARKIS, 2012). Como o GSCM não é um campo maduro, muitos pesquisadores o definiram de modo diferente (XU et al., 2013). Apesar de o tema de logística reversa ter recebido atenção nos últimos anos, é um conceito ainda relativamente novo e há poucas pesquisas sobre o tema (WIEL; BOSSINK; MASUREL, 2012).

O GSCM requer, além de atividades da cadeia de suprimentos direta adequadas ambientalmente, que tais atividades devem ser estendidas para incluir cadeias de suprimentos reversas de modo a implementar cadeias de suprimentos de "ciclo fechado" (closed-loop supply chains) (SARKIS, 2012). No "fechamento do ciclo" da cadeia, materiais em seus finais de vida são 
trazidos de volta ao sistema para serem reciclados, remanufaturados, reaproveitados, sendo a Logística Reversa somente parte do conceito de GSCM (SARKIS, 2012). A literatura indica os benefícios da implantação de práticas do GSCM, mas o meio empresarial ainda tem muitos desafios para estabelecer cadeias de suprimentos verdadeiramente sustentáveis, tendo que lidar com barreiras de implantação (RAUER; KAUFMAN, 2015). Mathiyazhagan et al. (2013) salientam que, durante a implementação do GSCM, as empresas precisam remover essas barreiras ou obstáculos. No entanto, observa-se que ainda há pouca avaliação sistematizada da literatura sobre essa questão. Diante dessa lacuna de pesquisa, o objetivo deste artigo é levantar na literatura, por meio da Revisão Bibliográfica Sistemática, as barreiras para a implantação efetiva da Logística Reversa e do GSCM.

O estudo conjunto do GSCM e da LR justifica-se pelos seguintes fatores:

- no decorrer do tempo, existência de muitas terminologias para GSCM (SARKIS, 2012; RAUER; KAUFMANN, 2015), incluindo termos sobre logística sustentável ou verde;

- histórico de confusão entre SCM e logística (com foco mais econômico) (CSCMP, 2017; LAMBERT; GARCÍA-DASTUGUE; CROXTON, 2008; LAMBERT, 2008; BOWERSOX et al., 2014; LAMBERT; COOPER, 2000; KAUFFMAN, 2002);

- LR como sendo a extensão da cadeia de suprimentos convencional para fechar o ciclo: tal extensão necessária para o GSCM (BEAMON, 1999; SARKIS, 2012; LINTON; KLASSEN; JAYARAMAN, 2007);

- LR como uma das áreas fundamentais para o processo de negócio "Gestão de Retornos" ou "Retorno" da SCM tradicional (modelo elaborado pelo GSCF - Global Supply Chain Forum - ou modelo SCOR) (LAMBERT; GARCÍA-DASTUGUE; CROXTON, 2008; LAMBERT, 2008);

- LR como uma área para a gestão efetiva dos resíduos gerados pela cadeia de suprimentos (LEITE, 2009).

Para tanto, na seção seguinte de Desenvolvimento, serão apresentados o método da pesquisa e o levantamento das barreiras à implementação efetiva da Logística Reversa e do GSCM. Por fim, serão exibidas as considerações finais.

\section{DESENVOLVIMENTO}

Esta seção tratará do método de pesquisa e da apresentação das barreiras ao GSCM e/ ou Logística Reversa.

\section{MÉTOdO DE PESQUISA}

Para a identificação de artigos sobre barreiras de implantação da Logística Reversa e do GSCM, foi realizada uma Revisão Bibliográfica Sistemática (RBS). A RBS utiliza procedimen- 
tos definidos para identificar, analisar e interpretar as evidências disponíveis relacionadas a uma questão de pesquisa ou fenômeno de interesse de forma imparcial, que possa ser reproduzível e ancorada em evidências (TRANFIELD; DENYER; SMART, 2003; LEVY; ELLIS, 2006; KITCHENHAM; CHARTERS, 2007).

As informações sobre a forma de condução da pesquisa estão detalhadas na Tabela 1.

Tabela 1 - Direcionamentos e resultados da busca.

\begin{tabular}{c|l}
\hline \multicolumn{2}{c}{ Direcionamentos de busca } \\
\hline Fenômeno de interesse & Barreiras de implantação da Logística Reversa e do GSCM \\
\hline Base de Dados & Web of Science \\
\hline Tempo de Busca & $1990-2016$ \\
\hline Termos de Busca & $\begin{array}{l}\text { ("critical success factors" ou "good practices" ou "barriers" e ("reverse } \\
\text { logistics" ou "green supply chain" ou "reverse supply chain" ou "closed } \\
\text { loop supply chain") }\end{array}$ \\
\hline Local de Busca & Título, resumo, palavras-chave e citações (referências) \\
\hline Número de artigos retornados & 79 \\
\hline $\begin{array}{c}\text { Número de artigos de periódicos } \\
\text { após leitura de título e resumo }\end{array}$ & 55 \\
\hline
\end{tabular}

Fonte: Elaborado pelas autoras.

A base de dados foi escolhida por ser relevante na área de interesse e por possuir abrangência internacional. A base é respeitada internacionalmente e apresenta periódicos indexados com avaliação de JCR (Journal Citation Reports), permitindo identificação do impacto do periódico.

Os termos de busca envolveram tanto palavras como barreiras (o principal interesse da busca), como boas práticas ou fatores críticos de sucesso (os últimos como sendo fatores requeridos para garantir o sucesso de determinada atividade) (LUTHRA et al., 2015). Como foi identificado que a ausência de fatores críticos ou boas práticas pode ser barreiras para a implantação, estes foram tratados como fatores similares, traduzidos como barreiras. Os termos "cadeia reversa de suprimentos" e "cadeia reversa de ciclo fechado" foram adicionados por poderem ser utilizados para LR ou GSCM.

Os artigos foram selecionados por intermédio de uma Revisão Sistemática na base de dados Web of Science. Após a identificação dos artigos por meio da string de busca (77 artigos identificados), estes foram filtrados para que apenas artigos publicados em periódicos (revistas) fossem investigados (55 artigos) por conta da relevância e abrangência, foram lidos todos os títulos, resumos e palavras-chave dos 55 artigos para a identificação daqueles que auxiliavam efetivamente na resposta à pergunta: Quais são as barreiras de implantação da Logística Reversa e do GSCM? A leitura foi realizada por dois pesquisadores, assim como, houve a dupla verificação de quais artigos deveriam ser ou não incluídos no estudo, sendo que foi utilizada uma abordagem conservadora de que se um pesquisador identificou contribuição o artigo seria lido na íntegra. Após essa etapa, foram selecionados 29 artigos, porém, três dos artigos não foram encontrados na íntegra, 26 foram lidos de forma completa e todas as barreiras de cada artigo 
foram selecionadas, novamente essa etapa foi feita por dois pesquisadores envolvidos. Foram adicionados dois artigos por cross-reference. Após a leitura completa, 25 artigos resultaram para compilação das barreiras de implantação. Os artigos são apresentados na Tabela 2.

A compilação das práticas em barreiras foi feita por meio da leitura e seleção das barreiras com dupla avaliação, com auxílio de tabelas para análise e compilação dos dados. As categorias e subcategorias foram criadas de acordo com o que foi encontrado nos artigos e já previamente dividido pelos autores selecionados.

Tabela 2 - Artigos selecionados por meio da RBS.

\begin{tabular}{|c|c|}
\hline Tema & Artigos \\
\hline Green Supply Chain Management & $\begin{array}{l}\text { Govindan et al. (2014); Li et al. (2015); Luthra et al. (2015); } \\
\text { Mathiyazhagan et al. (2013); Muduli et al. (2013a, b); Rauer e } \\
\text { Kaufmann (2015); Sarkis (2012); Wang et al. (2016); Wu et al. } \\
\text { (2015); Xu et al. (2013) }\end{array}$ \\
\hline Logística Reversa & $\begin{array}{l}\text { Abdulrahman et al. (2014); Bernon et al. (2013); Bouzon, Go- } \\
\text { vindan e Rodriguez (2015); Chileshe et al. (2015); Chiou et al. } \\
\text { (2012); Sencovici e Demajorovic (2015); González-Torre et al. } \\
\text { (2010); Hosseini et al. (2015); Kapetanopoulou e Tagaras (2011); } \\
\text { Prakash e Barua (2015); Pumpinyo e Nitivattananon (2013); Ravi } \\
\text { e Shankar (2005); Shaharudin, Zalani e Tan (2015); Wiel, Bossink } \\
\text { e Masurel (2012) }\end{array}$ \\
\hline
\end{tabular}

Fonte: Elaborado pelas autoras.

É perceptível identificar que o artigo mais antigo é de 2005 (Figura 1), havendo uma lacuna sobre o assunto (barreiras e fatores críticos) por quatro anos. O pico das publicações ocorreu em 2015. O baixo número de publicações de 2016 reflete o fato de a busca não ter englobado todo o ano de 2016 (foi finalizada em meados de 2016). Isso mostra que o tema é relevante e atual, necessitando de maior investigação.

Figura 1 - Distribuição de publicações por ano.

\section{Número de artigos por ano}

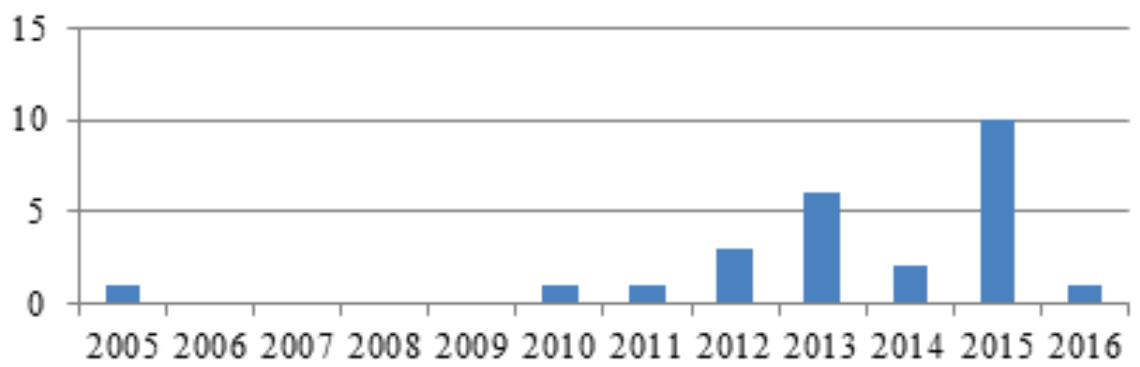

Fonte: Elaborado pelas autoras.

O principal objetivo da RBS foi a identificação das barreiras para a implantação da LR e do GSCM. A identificação das barreiras é de extrema importância, já que essa identificação 
pode ser tratada como um guia para tomar as ações apropriadas e superar os entraves de implantação da LR e do GSCM (RAVI; SHANKAR, 2005; MATHIYAZHAGAN et al., 2015; GOVINDAN et al., 2014). Há várias razões que influenciam as organizações na adoção de práticas de LR, mas a presença de barreiras torna a implementação difícil e barreiras distintas podem necessitar tratamentos e prioridades diferentes para um mesmo tipo de organização (PRAKASH; BARUA, 2015). Uma revisão da literatura é capaz de identificar vários tipos de barreiras que inibem a organização de ser ambientalmente adequada (MATHIYAZHAGAN et al., 2015). As barreiras serão exibidas e discutidas na seção seguinte.

As categorias foram escolhidas de acordo com o que já havia sido exposto e utilizado por autores que dividiam as barreiras em categorias, como os trabalhos de Abdulrahman Gunasekaran e Subramanian (2014), Govindan et al. (2014), Pumpinyo e Nitivattananon (2014), Xu et al. (2013). Após a análise das categorias identificadas, rearranjos foram realizados para a criação de subcategorias para a maior compreensão e melhor agrupamentos de práticas similares.

\section{BARREIRAS À IMPLEMENTAÇÃO EFETIVA DO GSCM E DA LOGÍSTICA REVERSA: RESULTA- DOS DA RBS}

As barreiras foram compiladas em sete diferentes categorias: gestão, financeiras, stakeholders, infraestrutura e tecnologia, ciclo de vida, conhecimento e cultura. As Tabelas 3 a 9 apresentam todas as 59 barreiras à implementação da LR e/ou GSCM, assim como, o seu enquadramento em categorias e suas subcategorias. As referências foram identificadas nas tabelas por meio de números, sendo apresentadas após a Tabela 9.

Tabela 3 - As barreiras de Gestão levantadas na literatura.

\begin{tabular}{|c|c|c|c|}
\hline \multicolumn{4}{|c|}{ Categoria: 1) Barreiras de Gestão } \\
\hline Subcategorias & & Barreiras & Referências \\
\hline Políticas Internas & 1 & $\begin{array}{l}\text { Falta de políticas organizacionais que apoiem a gestão ambiental } \\
\text { dentro da organização e ao longo da cadeia de suprimentos (GSCM) } \\
\text { e a logística reversa (LR) }\end{array}$ & $\begin{array}{l}1 ; 19 ; 16 ; 8 ; 13 ; \\
12 ; 4\end{array}$ \\
\hline $\begin{array}{l}\text { Comprometimento da } \\
\text { Alta Administração }\end{array}$ & 2 & $\begin{array}{l}\text { Falta de comprometimento efetivo da alta administração da empresa } \\
\text { na adoção do GSCM e/ou da LR }\end{array}$ & $\begin{array}{l}2 ; 2 ; 1 ; 4 ; 7 ; 14 ; 18 \\
19 ; 16 ; 2 ; 12 ; 8 ; 20 \\
13 ; 21 ; 5\end{array}$ \\
\hline \multirow{2}{*}{$\begin{array}{l}\text { Sistema de Medição de } \\
\text { Desempenho }\end{array}$} & 3 & $\begin{array}{l}\text { Falta de um apropriado sistema de medição de desempenho e re- } \\
\text { compensas ou de métricas para o GSCM e/ou LR }\end{array}$ & $16 ; 1 ; 14 ; 19 ; 2$ \\
\hline & 4 & $\begin{array}{l}\text { Indisponibilidade de dados para medir o desempenho do GSCM/ } \\
\text { LR e suas práticas }\end{array}$ & $18 ; 22$ \\
\hline $\begin{array}{l}\text { Planejamento } \\
\text { Estratégico }\end{array}$ & 5 & Falta de Planejamento Estratégico e planos de ação para a LR & $\begin{array}{l}1 ; 19 ; 16 ; 12 ; 3 ; 17 ; \\
21 ; 14 ; 2 ; 7\end{array}$ \\
\hline Estrutura Organizacional & 6 & $\begin{array}{l}\text { Falta de estrutura organizacional que seja adequada e incentive o } \\
\text { comprometimento de todos os níveis da empresa com o meio am- } \\
\text { biente e com a LR }\end{array}$ & $4 ; 7 ; 13 ; 2$ \\
\hline \multirow[t]{2}{*}{ Comunicação } & 7 & $\begin{array}{l}\text { Falta de transparência ou visibilidade ambiental decorrente da au- } \\
\text { sência de mecanismos de comunicação formais e informais: falta de } \\
\text { compartilhamento das informações ambientais internamente (entre } \\
\text { as áreas da empresa) e com clientes e fornecedores na cadeia de } \\
\text { suprimentos }\end{array}$ & $18 ; 8 ; 13 ; 2 ; 4 ; 14$ \\
\hline & 8 & Falta de qualidade das informações ambientais compartilhadas & $2 ; 12$ \\
\hline
\end{tabular}

Fonte: Elaborado pelas autoras. 
Tabela 4 - As barreiras financeiras levantadas na literatura.

\begin{tabular}{|c|c|c|c|}
\hline \multicolumn{4}{|c|}{ Categoria: 2) Barreiras Financeiras } \\
\hline Subcategorias & & Barreiras & Referências \\
\hline \multirow[b]{2}{*}{$\begin{array}{l}\text { Restrições Finan- } \\
\text { ceiras }\end{array}$} & 9 & $\begin{array}{l}\text { Possibilidade de altos investimentos para a im- } \\
\text { plantação de práticas ambientais e baixos/incertos } \\
\text { retornos econômicos }\end{array}$ & $\begin{array}{l}8 ; 20 ; 13 ; 16 ; 23 ; 3 ; 4 ; 7 ; \\
12 ; 10\end{array}$ \\
\hline & 10 & $\begin{array}{l}\text { Falta de reconhecimento da influência dos impac- } \\
\text { tos ambientais sobre o desempenho financeiro da } \\
\text { organização, assim a LR e outras práticas ambien- } \\
\text { tais não são uma prioridade de investimento nas } \\
\text { organizações }\end{array}$ & $5 ; 4 ; 1 ; 8$ \\
\hline Subcategorias & & Barreiras & Referências \\
\hline $\begin{array}{l}\text { Restrições Finan- } \\
\text { ceiras (cont.) }\end{array}$ & 11 & $\begin{array}{l}\text { Altos custos ou falta de recursos financeiros para } \\
\text { o GSCM, LR ou para tornar produtos e processos } \\
\text { ambientalmente adequados: tecnologias de processo } \\
\text { (máquinas, certificações), contratação e treinamento } \\
\text { de pessoal, para operacionalização dos processos } \\
\text { (coleta, transporte, armazenamento, manuseio dos } \\
\text { retornos, reprocessamento etc.), para implantação } \\
\text { de sistema de monitoramento dos retornos }\end{array}$ & $\begin{array}{l}1 ; 3 ; 8 ; 7 ; 19 ; 21 ; 17 ; 13 \\
4 ; 15 ; 11 ; 5 ; 18 ; 20 ; 23\end{array}$ \\
\hline $\begin{array}{l}\text { Restrições de Cré- } \\
\text { dito }\end{array}$ & 12 & $\begin{array}{l}\text { Indisponibilidade ou dificuldade para obter emprés- } \\
\text { timos bancários para implantar esforços de adequa- } \\
\text { ção ambiental }\end{array}$ & $13 ; 20 ; 8 ; 17 ; 4$ \\
\hline \multirow{3}{*}{ Altos Custos } & 13 & $\begin{array}{l}\text { Altos custos para operacionalização da LR (coleta, } \\
\text { transporte, reprocessamento) e estratégias de reva- } \\
\text { lorização (reuso, remanufatura e reciclagem) diante } \\
\text { do preço de revenda ou dos baixos custos relativos } \\
\text { para disposição final em aterros }\end{array}$ & $16 ; 4 ; 9 ; 5 ; 8 ; 11$ \\
\hline & 14 & $\begin{array}{l}\text { Falta de sistema de recompensas aos membros da } \\
\text { cadeia de suprimentos por suas práticas ambientais } \\
\text { e conflitos na divisão dos custos da coleta dos resí- } \\
\text { duos/materiais }\end{array}$ & $6 ; 2 ; 8$ \\
\hline & 15 & $\begin{array}{l}\text { Altos custos para destinação de substâncias perigo- } \\
\text { sas, devido aos riscos envolvidos }\end{array}$ & $8 ; 12 ; 13 ; 23$ \\
\hline
\end{tabular}

Fonte: Elaborado pelas autoras. 
Tabela 5 - As barreiras de Stakeholders levantadas na literatura.

\section{Categoria: 3) Barreiras de Stakeholders}

\begin{tabular}{|c|c|c|c|}
\hline \multirow[t]{3}{*}{ Subcategorias } & & Barreiras & Referências \\
\hline & 16 & $\begin{array}{l}\text { Falta de políticas governamentais de apoio para a LR } \\
\text { ou outras iniciativas organizacionais ambientalmente } \\
\text { corretas }\end{array}$ & $1 ; 4 ; 17 ; 23 ; 13 ; 20 ; 7 ; 16$ \\
\hline & 17 & $\begin{array}{l}\text { Impostos governamentais: falta de política de impostos } \\
\text { preferenciais, muitos tipos de impostos }\end{array}$ & $1 ; 6 ; 23 ; 17$ \\
\hline \multirow[t]{3}{*}{$\begin{array}{l}\text { Governo: Polí- } \\
\text { ticas e Impostos } \\
\text { governamentais, } \\
\text { Regulamentações } \\
\text { e legislação }\end{array}$} & 18 & $\begin{array}{l}\text { Leis e regulamentações ambientais governamentais ina- } \\
\text { dequadas: não específicas, com lacunas, não exequíveis, } \\
\text { mutáveis conforme política governamental, não fortes o } \\
\text { suficiente para forçar o comportamento ambientalmente } \\
\text { correto das empresas. Incongruências em regulamentações } \\
\text { ambientais entre os governos federal, estaduais e municipais }\end{array}$ & $\begin{array}{l}4 ; 7 ; 17 ; 23 ; 5 ; 3 ; 1 ; 9 ; 3 ; 8 ; \\
11 ; 16 ; 15 ; 12 ; 18\end{array}$ \\
\hline & 19 & $\begin{array}{l}\text { Falta de consciência sobre as regulamentações governa- } \\
\text { mentais }\end{array}$ & 1 \\
\hline & 20 & $\begin{array}{l}\text { Falta de fiscalização rigorosa de autoridades governa- } \\
\text { mentais no cumprimento da legislação. Falta de aplica- } \\
\text { ção das legislações }\end{array}$ & $15 ; 6 ; 1 ; 16 ; 18$ \\
\hline
\end{tabular}

\begin{tabular}{|c|c|c|c|}
\hline Subcategorias & & Barreiras & Referências \\
\hline \multirow{3}{*}{ Clientes } & 21 & $\begin{array}{l}\text { Retorno do produto pelo cliente: falta de informação sobre } \\
\text { formas de retorno, resistência para realizar o retorno ou } \\
\text { falta de programas e incentivos pelo governo ou empresas }\end{array}$ & $1 ; 7 ; 2 ; 4 ; 17 ; 12 ; 23$ \\
\hline & 22 & $\begin{array}{l}\text { Baixa demanda dos clientes por produtos eco-friendly, } \\
\text { devido à falta de consciência ambiental }\end{array}$ & $8 ; 13 ; 20 ; 15 ; 5 ; 12 ; 16$ \\
\hline & 23 & $\begin{array}{l}\text { Percepção de que os produtos/itens recuperados pos- } \\
\text { suem baixa qualidade }\end{array}$ & $4 ; 17 ; 12 ; 9 ; 7 ; 3 ; 19 ; 16$ \\
\hline \multirow{3}{*}{ Fornecedores } & 24 & $\begin{array}{l}\text { Resistência dos fornecedores para adotar iniciativas } \\
\text { ambientais }\end{array}$ & $8 ; 20 ; 23 ; 13 ; 18 ; 8$ \\
\hline & 25 & $\begin{array}{l}\text { Falta de influência sobre fornecedores de segunda cama- } \\
\text { da e outros níveis da cadeia de suprimentos }\end{array}$ & 18 \\
\hline & 26 & $\begin{array}{l}\text { Dificuldade em medir e monitorar as práticas ambientais } \\
\text { dos fornecedores }\end{array}$ & $8 ; 20 ; 13$ \\
\hline Concorrentes & 27 & $\begin{array}{l}\text { Ausência de competição no mercado ou pressões de con- } \\
\text { correntes que incentivem a adoção do GSCM ou LR }\end{array}$ & $1 ; 12 ; 17 ; 23 ; 5$ \\
\hline \multirow[t]{2}{*}{$\begin{array}{l}\text { Cadeia de Supri- } \\
\text { mentos }\end{array}$} & 28 & $\begin{array}{l}\text { Falta de envolvimento, colaboração, coordenação e in- } \\
\text { tegração dos membros da cadeia de suprimentos direta } \\
\text { (fornecedores, clientes, incluindo atacadistas e varejistas) } \\
\text { na adoção de práticas ambientais, incluindo a LR }\end{array}$ & $\begin{array}{l}12 ; 19 ; 22 ; 23 ; 1 ; 7 ; 4 ; 16 \\
3 ; 17\end{array}$ \\
\hline & 29 & $\begin{array}{l}\text { Falta de coordenação e integração entre a cadeia de } \\
\text { suprimentos direta e a cadeia reversa (LR) }\end{array}$ & 16 \\
\hline \multirow[t]{2}{*}{ Pressões Sociais } & 30 & $\begin{array}{l}\text { Falta de pressão social exercida por atores sociais (como } \\
\text { comunidade vizinha e sociedade, associações profissio- } \\
\text { nais, ONGs) para a adequação ambiental das empresas }\end{array}$ & $14 ; 7 ; 21 ; 4 ; 12$ \\
\hline & 31 & $\begin{array}{l}\text { Falta de incentivo das empresas para o envolvimento da } \\
\text { sociedade na adequação ambiental das empresas }\end{array}$ & $17 ; 12$ \\
\hline
\end{tabular}

Fonte: Elaborado pelas autoras.

78 REVISTA DE CIÊNCIA \& TECNOLOGIA • v. 20, N. 40, p. 69-88 • 2017• ISSN Impresso: 0103-8575• ISSN Eletrônico: ISSN: 2238-1252 
Tabela 6 - As barreiras de infraestrutura e de tecnologia levantadas na literatura.

\begin{tabular}{|c|c|c|c|}
\hline \multicolumn{3}{|r|}{ Categoria: 4) Barreiras de infraestrutura e de tecnologia } & \multirow[b]{2}{*}{ Referências } \\
\hline Subcategorias & & Barreiras & \\
\hline Infraestrutura & 32 & $\begin{array}{l}\text { Falta de infraestruturas próprias para LR (como local para armazenagem, } \\
\text { sistemas de transporte e computacionais, equipamentos e processos) }\end{array}$ & $1 ; 16 ; 3 ; 9 ; 7 ; 4$ \\
\hline \multirow[b]{2}{*}{$\begin{array}{l}\text { Tecnologia e Pro- } \\
\text { cesso }\end{array}$} & 33 & $\begin{array}{l}\text { Falta de novas tecnologias ou tecnologias apropriadas, materiais eco- } \\
\text { friendly e processos que possibilitem a implantação do GSCM }\end{array}$ & $\begin{array}{l}8 ; 20 ; 13 ; 15 ; 3 ; \\
17 ; 9 ; 7 ; 16\end{array}$ \\
\hline & 34 & $\begin{array}{l}\text { Existência de restrições técnicas para a implantação da LR ou falta de } \\
\text { tecnologias e instalações para a recuperação de produtos vindos da LR } \\
\text { e alta incerteza associada (incerteza no volume de material retornado } \\
\text { e falta de velocidade de reprocessamento desses materiais, o que pode } \\
\text { gerar um alto tempo médio de reprocessamento) }\end{array}$ & $\begin{array}{l}17 ; 4 ; 16 ; 1 ; \\
5 ; 3\end{array}$ \\
\hline Subcategorias & & Barreiras & Referências \\
\hline \multirow{2}{*}{$\begin{array}{l}\text { Sistemas e } \\
\text { Tecnologia da } \\
\text { Informação }\end{array}$} & 35 & $\begin{array}{l}\text { Falta de SI e TI que propiciem uma plataforma integrada efetiva para } \\
\text { o compartilhamento e a visibilidade de informações ambientais, intra e } \\
\text { interorganizacionais, para o GSCM e/ou LR }\end{array}$ & $\begin{array}{l}2 ; 4 ; 19 ; 16 ; 7 ; \\
3 ; 12 ; 5\end{array}$ \\
\hline & 36 & $\begin{array}{l}\text { Falta de segurança da informação e dos dados dentro da cadeia de supri- } \\
\text { mentos }\end{array}$ & 16 \\
\hline
\end{tabular}

Fonte: Elaborado pelas autoras.

Tabela 7 - As barreiras do Ciclo de Vida levantadas na literatura.

\begin{tabular}{|c|c|c|c|}
\hline \multicolumn{3}{|r|}{ Categoria: 5) Barreiras do Ciclo de Vida } & \multirow{3}{*}{$\begin{array}{l}\text { Referências } \\
1 ; 22 ; 8 ; 20 ; \\
13\end{array}$} \\
\hline Subcategorias & & Barreiras & \\
\hline \multirow[b]{2}{*}{ Ecodesign } & 37 & $\begin{array}{l}\text { Falta de utilização do Ecodesign voltado à escolha de materiais ambien- } \\
\text { talmente corretos }\end{array}$ & \\
\hline & 38 & $\begin{array}{l}\text { Falta de utilização de Ecodesign visando à fácil desmontagem do } \\
\text { produto para recuperar a quantidade máxima de materiais, visando à } \\
\text { estratégias de destinação do reuso, remanufatura e reciclagem }\end{array}$ & $9 ; 4 ; 7$ \\
\hline \multirow{2}{*}{$\begin{array}{l}\text { Recuperação: reu- } \\
\text { so, remanufatura e } \\
\quad \text { reciclagem }\end{array}$} & 39 & $\begin{array}{l}\text { Dificuldade na aplicação das estratégias de recuperação de produtos (reu- } \\
\text { so, remanufatura e reciclagem) devido às diferenças nas taxas de deterio- } \\
\text { ração e na qualidade e limpeza dos materiais extraídos, à dificuldade de } \\
\text { identificação dos materiais e à existência de substâncias perigosas }\end{array}$ & $4 ; 9 ; 17 ; 22$ \\
\hline & 40 & $\begin{array}{l}\text { Falta de mercado e baixo valor econômico para produtos recuperados no } \\
\text { fim da vida útil }\end{array}$ & $3 ; 16$ \\
\hline \multirow{2}{*}{$\begin{array}{l}\text { Logística e Canais } \\
\text { de retorno }\end{array}$} & 41 & $\begin{array}{l}\text { Falta de informações sobre mapeamento dos agentes dos canais de re- } \\
\text { torno, como localização dos pontos de origem do sistema de LR, identi- } \\
\text { ficação de parceiros e prestadores de serviço }\end{array}$ & $3 ; 1 ; 17 ; 4 ; 9$ \\
\hline & 42 & $\begin{array}{l}\text { Dificuldades para a terceirização da operação da LR: complexidade na } \\
\text { identificação, estabelecimento de parceria e coordenação de Operadores } \\
\text { Logísticos terceirizados especializados em LR }\end{array}$ & $1 ; 16 ; 8 ; 20$ \\
\hline
\end{tabular}

Fonte: Elaborado pelas autoras. 
Tabela 8 - As barreiras de Conhecimento levantadas na literatura.

\begin{tabular}{|c|c|c|c|}
\hline \multicolumn{3}{|r|}{ Categoria: 6) Barreiras de Conhecimento } & \multirow[b]{2}{*}{ Referências } \\
\hline Subcategorias & & Barreiras & \\
\hline \multirow{3}{*}{$\begin{array}{l}\text { Recursos Hu- } \\
\text { manos }\end{array}$} & 43 & $\begin{array}{l}\text { Falta de recursos humanos em quantidade e com qualifi- } \\
\text { cação técnica e gerencial necessária para a implantação de } \\
\text { sistemas de gestão ambiental, como LR e GSCM }\end{array}$ & $\begin{array}{l}4 ; 7 ; 15 ; 13 ; 14 ; 17 ; 3 \\
20 ; 8 ; 16 ; 17\end{array}$ \\
\hline & 44 & $\begin{array}{l}\text { Falta de especialistas em gestão da LR ou GSCM para } \\
\text { elaboração de projeto na busca de financiamento }\end{array}$ & $1 ; 17 ; 23 ; 8$ \\
\hline & 45 & $\begin{array}{l}\text { Falta de motivação e comprometimento dos funcionários } \\
\text { da organização na adoção do GSCM e LR e seus novos } \\
\text { sistemas e tecnologias }\end{array}$ & $15 ; 2 ; 7 ; 8$ \\
\hline \multirow[t]{2}{*}{ Treinamento } & 46 & $\begin{array}{l}\text { Falta de participação dos funcionários em programas de } \\
\text { educação ambiental (como seminários, reuniões) condu- } \\
\text { zidos pelo governo ou organizações de referência que } \\
\text { adotaram com sucesso os conceitos de gestão ambiental } \\
\text { como GSCM }\end{array}$ & $8 ; 13$ \\
\hline & 47 & $\begin{array}{l}\text { Falta de programas de educação ambiental e treinamen- } \\
\text { tos internos à organização e relacionados ao GSCM e/ou } \\
\text { LR e falta de instituições como consultorias para treinar, } \\
\text { monitorar e orientar o progresso }\end{array}$ & $3 ; 19 ; 4 ; 13 ; 8 ; 20$ \\
\hline \multirow{4}{*}{ Conhecimento } & 48 & $\begin{array}{l}\text { Falta de conhecimento ambiental (inclusive de legislações } \\
\text { ambientais) pelos membros da cadeia de suprimentos } \\
\text { para a implantação do GSCM }\end{array}$ & $8 ; 12 ; 8 ; 13 ; 14 ; 15$ \\
\hline & 49 & $\begin{array}{l}\text { Falta de entendimento sobre as práticas do GSCM e/ou } \\
\text { LR e ausência de padronização dos seus processos }\end{array}$ & $\begin{array}{l}8 ; 1 ; 4 ; 16 ; 19 ; 7 ; 13 ; 20 ; \\
3 ; 21\end{array}$ \\
\hline & 50 & $\begin{array}{l}\text { Falta de reconhecimento sobre a vantagem competitiva } \\
\text { na adoção de estratégias Green e de LR }\end{array}$ & $23 ; 5 ; 3 ; 8 ; 8$ \\
\hline & 51 & $\begin{array}{l}\text { Falta de conhecimento sobre tributação dos produtos } \\
\text { retornados }\end{array}$ & 3 \\
\hline Conscientização & 52 & $\begin{array}{l}\text { Falta de consciência e confiança nos benefícios para a } \\
\text { organização advindos do GSCM e/ou LR }\end{array}$ & $4 ; 3 ; 8 ; 13 ; 20$ \\
\hline
\end{tabular}

Fonte: Elaborado pelas autoras.

Tabela 9 - As barreiras de Cultura levantadas na literatura.

\begin{tabular}{c|c|l|l}
\hline \multicolumn{1}{c}{ Cubcategorias } & 53 & $\begin{array}{l}\text { Categoria: 7) Barreira de Cultura } \\
\text { Predomínio de população cuja cultura não valoriza a preser- } \\
\text { vação ambiental e sistemas ambientais como a LR }\end{array}$ & Referências \\
\cline { 2 - 4 } Cultura & 54 & $\begin{array}{l}\text { Falta de cultura organizacional que motive os funcionários } \\
\text { no engajamento da preservação ambiental e na realização de } \\
\text { melhorias ambientais }\end{array}$ & 14 \\
\cline { 2 - 5 } & 55 & $\begin{array}{l}\text { Cultura organizacional ancorada na falta de confiança entre } \\
\text { a alta gerência e os empregados, ocasionando falta de con- } \\
\text { fiança, comunicação e integração interorganizacional entre } \\
\text { os funcionários das empresas da cadeia de suprimentos para } \\
\text { a preservação ambiental }\end{array}$ & 14 \\
\hline
\end{tabular}

continua 
continuação

\begin{tabular}{c|c|l|c|c}
\hline Subcategorias & \multicolumn{2}{c}{ Barreiras } & \multicolumn{2}{c}{ Referências } \\
\cline { 2 - 4 } Resistência & 56 & $\begin{array}{l}\text { Resistência à mudança pelos colaboradores quando da adoção de } \\
\text { sistemas ambientais como LR e GSCM }\end{array}$ & $\begin{array}{l}14 ; 2 ; 19 ; 17 ; \\
18 ; 4 ; 16 ; 21\end{array}$ \\
\cline { 2 - 5 } & 57 & $\begin{array}{l}\text { Medo do fracasso em adotar novos sistemas, como o GSCM } \\
\text { devido ao medo de perdas monetárias, falhas no produto e, por } \\
\text { conseguinte, perda de vantagem competitiva }\end{array}$ & $8 ; 20 ; 13$ \\
\hline \multirow{2}{*}{$\begin{array}{c}\text { Responsabilida- } \\
\text { de Ambiental e } \\
\text { Social }\end{array}$} & 58 & $\begin{array}{l}\text { Falta de percepção da responsabilidade ambiental: a organização } \\
\text { acredita que tomar medidas ambientais não é sua responsabilidade }\end{array}$ & $8 ; 13$ \\
\cline { 2 - 5 } & 59 & $\begin{array}{l}\text { Falta de Responsabilidade Social por parte das empresas: conse- } \\
\text { quências públicas das ações ambientais }\end{array}$ & $8 ; 13 ; 5$ \\
\hline
\end{tabular}

Fonte: Elaborado pelas autoras.

As referências e respectivas numerações são:

1) Abdulrahman Gunasekaran e Subramanian (2014); 2) Bernon et al. (2012); 3) Bouzon, Govindan e Rodrigues (2016); 4) Chileshe, Rameezdeen e Lehmann (2015); 5) Chiou et al. (2012); 6) Secovici e Demajorovic (2015); 7) González-Torre et al. (2010); 8) Govindan et al. (2014); 9) Hosseini et al. (2015); 10) Kapetanopoulou e Tagaras (2011); 11) Li et al. (2015); 12) Luthra et al. (2015); 13) Mathiyazhagan et al. (2013); 14) Muduli et al. (2013a); 15) Muduli et al. (2013b); 16) Prakash e Barua (2015); 17) Pumpinyo e Nitivattananon (2014); 18) Rauer e Kaufmann (2015); 19) Ravi e Shankar (2005); 20) Wang et al. (2016); 21) Wiel, Bossink e Masurel (2012); 22) Wu et al. (2015); 23) Xu et al. (2013).

As barreiras de gestão relacionam-se à ausência de elementos como política interna que apoie o GSCM e/ou LR e comprometimento da alta administração, planejamento estratégico da LR, estrutura organizacional que incentive o comprometimento dos funcionários com o meio ambiente e a LR. Outros elementos se referem ao compartilhamento de informações ambientais internamente e ao longo da cadeia de suprimentos e à medição de desempenho para GSCM e LR.

Políticas internas restritivas são barreiras importantes para a adoção e para a conscientização da importância da logística reversa. Uma dessas políticas é o não comprometimento da qualidade do produto final com a utilização de produtos retornados, não havendo a recuperação do valor secundário implícito no produto devolvido (ROGERS; TIBBEN-LEMBKE, 1998; RAVI; SHANKAR, 2005; PRAKASH; BARUA, 2015). Devido à responsabilidade estendida do produto, as empresas começaram a inserir opções de recuperação de produtos usados em sua estrutura de cadeia de suprimentos para recuperar o valor econômico que pode lhe dar vantagem sobre os concorrentes (RAVI; SHANKAR, 2005). O compromisso da alta administração é necessário para alocar recursos tecnológicos, financeiros e humanos adequados para a adoção de conceitos, ideias e desenvolvimento de estratégia para o GSCM (LUTHRA et al., 2015). Ainda há falta do envolvimento da alta administração na adoção da gestão da cadeia de suprimentos na forma de resistência da alta administração em mudar os 
investimentos, informações, sistemas e hábitos existentes (GOVINDAN et al., 2014; WANG et al., 2016; MATHIYAZHAGAN et al., 2013). Uma liderança eficaz é necessária para fornecer uma visão clara e valor para o programa de logística reversa (RAVI; SHANKAR, 2005). A alta administração também deve fornecer suporte contínuo para logística reversa nos planos estratégicos e nos planos de ação para implementá-la com sucesso (RAVI; SHANKAR, 2005; ABDULRAHMAN; GUNASEKARAN; SUBRAMANIAN, 2014). Devido às rápidas mudanças na tecnologia e nos comportamentos dos concorrentes, consumidores, fornecedores, e à pressão da sociedade e do governo, entre outros, um planejamento estratégico é necessário para os programas de logística reversa, GSCM e qualquer novo conceito de gestão organizacional, de forma a identificar metas e os planos de longo prazo (RAVI; SHANKAR, 2005; MUDULI et al., 2013a).

A medição de desempenho adequada de qualquer sistema é um elemento-chave para medir, documentar, controlar e melhorar o desempenho da logística reversa (RAVI; SHANKAR, 2005; PRAKASH; BARUA, 2015). Wu et al. (2015) salientam que a medição deve ser feita em relação aos impactos das práticas ambientais do GSCM, como, por exemplo, ter indicadores de operação relacionado ao uso de recursos e energia, emissões e redução do desperdício/ disposição final.

Apesar de as empresas estarem interessadas na economia gerada pela recuperação de produtos usados (RAVI; SHANKAR, 2005), há barreiras financeiras que são críticas à implantação da Logística Reversa e GSCM (ROGERS; TIBBEN-LEMBKE, 1998; ABDULRAHMAN; GUNASEKARAN; SUBRAMANIAN, 2014; GOVINDAN et al., 2014). Tais barreiras se relacionam aos seguintes elementos: possibilidade de altos investimentos e incertos retornos econômicos na adoção de práticas ambientais como GSCM e LR, altos custos para operacionalizar a LR e em especial a de substâncias perigosas, falta de alocação de recursos financeiros para tais práticas, dificuldade em obter empréstimos bancários, impasses na divisão de recompensas e custos relativos à LR pelos agentes da cadeia direta ou reversa. Em relação aos custos, muitas vezes, os baixos custos de descarte de materiais em aterros não justificam os custos da LR (CHILESHE et al., 2015; HOSSEINI et al., 2015; LI et al., 2015): custos de transporte de retorno, de remanufatura ou reciclagem e o preço de revenda (CHIOU et al., 2012). O tempo, a qualidade e a quantidade de retorno dos produtos são incertos, o que reduz a obtenção de economias de escala (PRAKASH; BARUA, 2015).

As barreiras de stakeholders incluem barreiras externas à empresa e englobam os seguintes elementos: muitos impostos e não sendo preferenciais, regulamentações ambientais inadequadas (com lacunas e não exequíveis) e falta de fiscalização e cumprimento, políticas governamentais de apoio à LR e outras práticas ambientais, resistência do cliente para realizar o retorno e mentalidade de que produtos recuperados são de qualidade inferior, resistência dos fornecedores e dificuldade no monitoramento das práticas ambientais dos fornecedores, falta de integração dos membros da cadeia de suprimentos direta (fornecedores, clientes, incluindo atacadistas e varejistas) para o GSCM e LR, falta de integração entre as cadeias direta e reversa, falta de pressão dos atores sociais. Sobre tributação, há falta de política fiscal preferencial para empresas que realizam a logística reversa e ações ambientalmente corretas (ABDULRAH- 
MAN; GUNASEKARAN; SUBRAMANIAN, 2014), há a cobrança de vários tipos de impostos (PUMPINYO; NITIVAT'TANANON, 2014) e a ausência de incentivos como a diminuição da taxação de produtos retornados (BOUZON; GOVINDAN; RODRIGUES, 2016).

Outro stakeholder importante para a adoção de ações ambientais é o cliente, que está começando a preferir comprar de organizações com consciência ambiental, portanto há um encorajamento e suporte dos clientes para ações ambientais e produtos verdes, que impulsionam a competitividade e a imagem da empresa tanto em mercados nacionais como internacionais (LUTHRA et al., 2015). Os fornecedores são stakeholders imprescindíveis para a implantação do GSCM, sendo que uma das dificuldades é que há problemas para manter fornecedores ambientalmente engajados, não havendo garantias de permanência do fornecedor e de parcerias de longo prazo (GOVINDAN et al., 2014; WANG et al., 2016). A resistência de engajamento ambiental também é refletida na falta de compromisso dos fornecedores em compartilhar informações sobre questões ambientais (GOVINDAN et al., 2014; MATHIYAZHAGAN et al., 2013), dificultando a medição e monitoramento das práticas ambientais realizadas por eles (MATHIYAZHAGAN et al., 2013; WANG et al., 2016).

Sobre as barreiras de infraestrutura e de tecnologia, a infraestrutura possui um papel vital na implantação da LR e barreiras envolvem a falta de instalações internas para armazenagem, transporte e falta de equipamentos e sistemas. Tecnologias para reciclagem aumentam o sucesso da implantação da LR, porém falta estrutura tecnológica (novas tecnologias, materiais e processos apropriados) para a adoção da LR e GSCM, o que afeta a capacidade de processamento de materiais retornados e o tempo médio de reciclagem (MUDULI et al., 2013a; ABDULRAHMAN; GUNASEKARAN; SUBRAMANIAN, 2014; PRAKASH; BARUA, 2015; BOUZON; GOVINDAN; RODRIGUES, 2016; GOVINDAN et al., 2014; WANG et al., 2016; MATHIYAZHAGAN et al., 2013; PUMPINYO; NITIVATTANANON, 2014; CHILESHE; RAMEEZDEEN; LEHMANN, 2015). SI e TI são importantes para o compartilhamento de informações ambientais dentro da organização e ao longo da cadeia de suprimentos.

No que tange às barreiras do Ciclo de Vida, elas envolvem elementos como: não adoção do Ecodesign para o auxílio da escolha de materiais e a desmontagem dos produtos, recuperação dificultada pelas diferenças nos materiais constituintes do produto, falta de mercado e baixo valor econômico para produtos recuperados, falta de mapeamento dos agentes do canal reverso e terceirização da LR. Para a sustentação da LR e GSCM, o ciclo de vida do produto precisa ser pensado de forma a ser ambientalmente correto, o projeto do produto deve ser realizado de modo a pensar no fim da vida útil do produto (ABDULRAHMAN; GUNASEKARAN; SUBRAMANIAN, 2014; WU et al., 2015), de maneira a desenvolver projetos de produtos que sejam recicláveis ou reutilizáveis (GOVINDAN et al., 2014; WANG et al., 2016; MATHIYAZHAGAN et al., 2013).

Para que seja possível a reciclagem ou reutilização, é necessário que haja uma fácil desmontagem do produto (HOSSEINI et al., 2015; CHILESHE; RAMEEZDEEN; LEHMANN, 2015; GONZÁLEZ-TORRE et al., 2010). A desmontagem é uma etapa complexa porque há dificuldade na identificação dos materiais (GONZÁLEZ-TORRE et al., 2010), incerteza do uso dos itens recuperados e existência de diferenças significativas na qualidade 
dos materiais extraídos (CHILESHE; RAMEEZDEEN; LEHMANN, 2015). Há, portanto, uma dificuldade tanto na desmontagem quanto na recuperação de produtos usados (WU et al., 2015). O baixo investimento pode ser resultado da falta de mercados/marketing para produtos recuperados e remanufaturados (BOUZON; GOVINDAN; RODRIGUES, 2016; PRAKASH, BARUA, 2015) e do baixo valor econômico recuperado de produtos no fim da vida (PRAKASH, BARUA, 2015).

Sobre as barreiras de Conhecimento, inserem-se elementos como recursos humanos especialistas em GSCM e LR, comprometimento dos funcionários na adoção do GSCM e LR, participação de funcionários em programas de educação ambiental ofertados pelo governo, pelas organizações de referência ou pela própria organização e falta de conhecimentos ambientais pelos membros da cadeia. Recursos humanos são necessários para a implantação da GSCM. Para isso, é necessário que haja práticas de gestão dos recursos humanos, de modo a possibilitar que as pessoas se sintam incentivadas a promover práticas ambientais. Uma das formas de envolver os recursos humanos é por meio de grupos de trabalho com foco ambiental, que possibilita o trabalho conjunto, voluntário e com indivíduos de diversos níveis hierárquicos, para encontrar soluções para problemas ambientais complexos (MUDULI et al., 2013b; LUTHRA et al., 2015).

Outra barreira é a falta de reconhecimento sobre a vantagem competitiva na adoção de estratégias Green e de LR. O GSCM é uma maneira de diferenciar uma empresa de seus concorrentes e pode influenciar a obtenção de sucesso (LUTHRA et al., 2015). Apesar de haver pouco reconhecimento de que a Logística Reversa seja um fator competitivo (ABDULRAHMAN; GUNASEKARAN; SUBRAMANIAN, 2014), alguns autores afirmam que as empresas estão utilizando suas capacidades de logística reversa e de estratégias verdes para fortalecer sua vantagem competitiva (CHIOU et al., 2012; XU et al., 2012).

Sobre as barreiras de Cultura, ressaltam-se os seguintes elementos: culturas nacional e organizacional que não valorizam a preservação ambiental, resistência dos funcionários na adoção do GSCM e LR, falta de responsabilidade ambiental das organizações e medo em perdas monetárias e de vantagem competitiva. A motivação para esforços de melhoria ambiental pode ser suportada ou minada pela cultura organizacional, mas uma cultura que encoraja a participação e estabelece respeito mútuo provavelmente aumentará o envolvimento dos colaboradores na realização de melhorias ambientais (MUDULI et al., 2013a). A resistência à mudança, que é uma barreira intrínseca à natureza humana, é um dos fatores críticos para a implantação de sistemas como LR e GSCM (MUDULI et al., 2013a; BERNON et al., 2012; RAVI; SHANKAR, 2005; PUMPINYO; NITIVATTANANON, 2014; RAUER; KAUFMANN, 2015). O medo de perdas monetárias também pode gerar resistência à mudança para o sistema de LR e GSCM (GOVINDAN et al., 2014; WANG et al., 2016; MATHIYAZHAGAN et al., 2013).

\section{CONSIDERAÇÕES FINAIS}

Este artigo buscou explorar os fatores que podem limitar a implantação efetiva dos conceitos de GSCM e/ou LR, identificando e sistematizando diversas barreiras presentes na li- 
teratura. Foram encontradas 59 barreiras, enquadradas em sete diferentes categorias: gestão (8 barreiras), financeiras (7), stakeholders (16), infraestrutura e tecnologia (5), ciclo de vida (6), conhecimento (10) e cultura (7). Assim, a literatura indica uma ampla variedade de fatores (de diversas naturezas) que podem dificultar a implementação de ações ambientalmente sustentáveis pelas organizações, em especial os conceitos de GSCM e LR. Destaca-se o alto número de barreiras relativas aos stakeholders, indicando que os agentes do ambiente compreendido pela cadeia de suprimentos, como fornecedores, clientes, concorrentes, inclusive governo e sociedade podem criar dificuldades a tal implementação devido às suas ações ou à falta delas.

Ressalta-se que esse levantamento abrangente envolve possíveis barreiras ao GSCM e LR, que, ao serem identificadas, podem ser pontos de reflexão e atenção de empresas que possuem o intuito de implantar ou melhorar sua gestão da cadeia de suprimentos focando a sustentabilidade ou a Logística Reversa. O levantamento das barreiras permite que a organização identifique quais estão presentes em sua realidade e elaborar planos de ação para saná-las, podendo atribuir diferentes graus de prioridade a cada uma.

O levantamento realizado também permite o embasamento de pesquisas futuras que tenham o intuito de avaliar nas organizações os impactos da existência de barreiras específicas, ou mesmo verificar, do ponto de vista de especialistas ou levantamentos em organizações, quais são as barreiras mais limitantes para a implantação do GSCM e LR. Em pesquisas futuras é possível realizar comparações entre o que foi identificado na base internacional (Web of Science) com bases nacionais e outras fontes, como congressos, para comparações e análise da pesquisa nacional. A maior parte da literatura não avalia as barreiras à implementação do GSCM para determinado setor empresarial (MATHIYAZHAGAN et al., 2013), sendo a pesquisa em diferentes setores específicos mais um ponto de avanço de conhecimento na área. Além do fator setorial, pesquisas futuras podem avaliar a influência de outros fatores contextuais (características internas das organizações e de seu ambiente) na determinação das barreiras e dificuldades existentes.

\section{RefERÊNCIAS}

ABDULRAHMAN, M. D.; GUNASEKARAN, A.; SUBRAMANIAN, N. "Critical barriers in implementing reverse logistics in the Chinese manufacturing sectors." International Journal of Production Economics, v. 147, p. 460-471, 2014.

BEAMON, B. M. "Designing the green supply chain." Logistics Information Management, v. 12, n. 4, 1999.

BERNON, M. et al. "An exploration of supply chain integration in the retail product returns process." International Journal of Physical Distribution \& Logistics Management, v. 43, n. 7, p. 586-608, 2013.

BOUZON, M.; GOVINDAN, K.; RODRIGUEZ, C. M. T. "Reducing the extraction of minerals: Reverse logistics in the machinery manufacturing industry sector in Brazil using ISM 
approach.” Resources Policy, v. 46, p. 27-36, 2015.

BOWERSOX, D. J. et al. Gestão Logística da Cadeia de Suprimentos. Porto Alegre: Bookman, Mc Graw Hill, 2014.

CHILESHE, N. et al. "Barriers to implementing reverse logistics in South Australian construction organizations.” Supply Chain Management: An International Journal, v. 20, n. 2, 179-204, 2015.

CHIOU, C. Y. et al. "Consideration factors of reverse logistics implementation-A case study of Taiwan's electronics industry." Procedia-Social and Behavioral Sciences, v. 40, p. 375381, 2012.

CSCMP (Council of Supply Chain Management Professionals) CSCMP Supply Chain Management Definitions. Disponível em: <www.cscmp.org>, acesso em: 14/11/2017.

GONZÁLEZ $\square$ TORRE, P. et al. "Barriers to the implementation of environmentally oriented reverse logistics: Evidence from the automotive industry sector." British Journal of Management, v. 21, n. 4, p. 889-904, 2010.

GOVINDAN, K. et al. "Barriers analysis for green supply chain management implementation in Indian industries using analytic hierarchy process." International Journal of Production Economics, v. 147, p. 555-568, 2014.

HOSSEINI, M. R. et al. "Reverse logistics in the construction industry." Waste Management \& Research, v. 33, n. 6, 499-514, 2015.

KAPETANOPOULOU, P.; TAGARAS, G. "Drivers and obstacles of product recovery activities in the Greek industry." International Journal of Operations \& Production Management, v. 31, n. 2, 148-166, 2011.

KAUFFMAN, R. G. "Supply Management: What's in a Name? Or, Do We Know Who We Are?" The Journal of Supply Chain Management, v. 38, n. 4, p. 46-50, nov., 2002.

KITCHENHAM, B. A.; CHARTERS, S. Procedures for Performing Systematic Literature Reviews in Software Engineering. Keele University \& Durham University, UK, 2007.

LAMBERT, D. M. Supply Chain Management: processes, partnerships, performance. Supply Chain Management Institute. EUA, 3. ed., 2008.

LAMBERT, D. M.; COOPER, M. C. “Issues in Supply Chain Management.” Industrial Marketing Management, New York, v. 29, p. 65-83, 2000.

LAMBERT, D. M.; GARCÍA-DASTUGUE, S. J.; CROXTON, K. L. "The role of logistics managers in the cross-functional implementation of supply chain management.” Journal of Business Logistics, v. 29, n. 1, 2008.

LEITE, P. R. Logística Reversa: meio ambiente e competitividade. São Paulo: Pearson Prentice Hall, 2. ed., 2009. 
LEVY, Y.; ELLIS, T. J. "A systems approach to conduct an effective literature review in support of information systems research." Informing Science: International Journal of an Emerging Transdiscipline, v. 9, n. 1, p. 181-212, 2006.

LI, J.; PAN, S. Y.; KIM, H.; LINN, J. H.; CHIANG, P. C. "Building green supply chains in ecoindustrial parks towards a green economy: Barriers and strategies." Journal of environmental management, v. 162, p. 158-170, 2015.

LINTON, J. D.; KLASSEN, R.; JAYARAMAN, V. "Sustainable supply chains: an introduction.” Journal of Operations Management, v. 25, 2007.

LUTHRA, S. et al. "Barriers to renewable/sustainable energy technologies adoption: Indian perspective." Renewable and Sustainable Energy Reviews, v. 41, p. 762-776, 2015.

MATHIYAZHAGAN, K. et al. "An ISM approach for the barrier analysis in implementing green supply chain management.” Journal of Cleaner Production, v. 47, p. 283-297, 2013.

MUDULI, K. et al. "Barriers to green supply chain management in Indian mining industries: a graph theoretic approach.” Journal of Cleaner Production, v. 47, p. 335-344, 2013 b.

MUDULI, K. et al. "Role of behavioural factors in green supply chain management implementation in Indian mining industries." Resources, Conservation \& Recycling, v. 76, p. 50-60, 2013a.

OECD (Organisation for Economic Co-operation and Development). About the OECD. Disponível em: http://www.oecd.org. Acesso em 09/03/2016.

OECD (Organisation for Economic Co-operation and Development, OECD Guidelines for multinational enterprises. Edição 2011. OECD Publishing. Disponível em: http://dx.doi. org/10.1787/9789264115415-en. Acesso em 09/03/2016.

POLÍTICA NACIONAL DE RESÍDUOS SÓLIDOS (PNRS). Lei 12.305, de 2 de agosto de 2010. Disponível em: http://www.planalto.gov.br/ccivil 03/ ato2007-2010/2010/lei/ 112305.htm. Acesso em: 15/03/2016.

PRAKASH, C.; BARUA, M. K. "Integration of AHP-TOPSIS method for prioritizing the solutions of reverse logistics adoption to overcome its barriers under fuzzy environment." Journal of Manufacturing Systems, v. 37, p. 599-615, 2015.

PUMPINYO, S.; NITIVATTANANON, V. "Investigation of Barriers and Factors Affecting the Reverse Logistics of Waste Management Practice: A Case Study in Thailand." Sustainability, v. 6, p. 7.048-7.062, 2014.

RAUER, J.; KAUFMANN, L. "Mitigating external barriers to implementing Green Supply Chain Management: a grounded theory investigation of green-tech companies'rare earth metals supply chains." Journal of Supply Chain Management, v. 51, n. 2, 2015.

RAVI, V.; SHANKAR, R. "Analysis of interactions among the barriers of reverse logistics." Technological Forecasting \& Social Change, v. 72, p. 1.011-1.029, 2005. 
SARKIS, J. "A boundaries and flows perspective of green supply chain management." Supply Chain Management: An International Journal, v. 17, n. 2 p. 202-216, 2012.

SENCOVICI, L. A.; DEMAJOROVIC, J. "Barriers and Prospects for Reverse Logistics as Regards oil Lube and its Packaging." Revista de Gestão Ambiental e Sustentabilidade, v. 4, n. 2, p. 83-101, 2015.

SHAHARUDIN, M. R.; ZAILANI, S.; TAN, K. C. "Barriers to product returns and recovery management in a developing country: investigation using multiple methods." Journal of Cleaner Production, v. 96, p. 220-232, 2015.

TRANFIELD, D; DENYER, D.; SMART, P. “Towards a methodology for developing evidence-informed management knowledge by means of systematic review." British Journal of Management, v. 14, n. 3, p. 207-222, 2003.

WANG, Z. et al. "A decision making trial and evaluation laboratory approach to analyze the barriers to Green Supply Chain Management adoption in a food packaging company." Journal of Cleaner Production, v. 117, p. 19-28, 2016.

WIEL, A. V., BOSSINK, B.; MASUREL, E. "Reverse logistics for waste reduction in cradleto-cradle-oriented firms: waste management strategies in the Dutchmetal industry." International Journal of Technology Management, v. 60, n. 1/2, p. 96-113, 2012.

WU, K. et al. "Exploring decisive factors in green supply chain practices under uncertainty." International Journal of Production Economics, v. 159, p. 147-157, 2015.

XU, L. et al. "Multiple comparative studies of Green Supply Chain Management: Pressures analysis.” Resources, Conservation and Recycling, v. 78, p. 26-35, 2013.

Submetido em: 15-11-2017

Aceito em: 20-3-2018 